\title{
Estimación del aprendizaje matemático mediante la versión española del Test de Evaluación Matemática Temprana de Utrecht
}

\author{
José I. Navarro, Manuel Aguilar, Concepción Alcalde, Esperanza Marchena, \\ Gonzalo Ruiz, Inmaculada Menacho y Manuel G. Sedeño \\ Universidad de Cádiz (España)
}

El Test de Evaluación Matemática Temprana está orientado a medir el nivel de competencia matemática temprana. Se ha desarrollado para de educación infantil y $1^{\circ} \mathrm{y}$ $2^{\circ}$ de primaria. Dispone de tres versiones paralelas, de 40 ítems cada una. Consta de 8 tareas, divididas en grupos de 5: Comparación, clasificación, correspondencia, seriación, conteo verbal, conteo estructurado, conteo resultante y conocimiento general de los números. Tiene una puntuación máxima de 40 puntos (uno por cada ítem correcto). El objetivo fundamental de este estudio consistió en adaptar el test a la población española con todas las garantías de validez y fiabilidad, que permita tener un instrumento para detectar posibles problemas en el aprendizaje de las matemáticas en los inicios de la escolaridad. El TEMT se ha baremado con una muestra de 1.053 niños/as pertenecientes a 14 colegios. 539 eran varones y 514 mujeres. Nos da un índice cuantitativo del nivel de competencia matemática temprana, posee un buen valor predictivo sobre el rendimiento matemático y los valores de validez y fiabilidad obtenidos cumplen con los criterios estándar.

Palabras clave: Matemática temprana, cognición, evaluación, educación infantil, educación primaria, sentido numérico.

\begin{abstract}
Assessment of math learning by the Early Mathematical Competence Test Spanish version. The Early Mathematical Competence Test (EMTC) assesses mathematical knowledge for kindergarden and primary school children. The test has 3 versions, of 40 items each and measures eight aspects of mathematical competence: Concepts of comparison; Classification; Correspondence; Seriation; Using counting words, forwards and backwards; Structures counting; Resultative counting; and General knowledge of numbers. Each subtest has five questions and a total score of 40 points. The main aim of this study was the standardization of EMTC for a Spanish population, following the international reliability and validation statistical rules. The test was administered to 1053 participants aged 4 to 7 (539 male and 514 female) from 14 schools. The EMTC provides a Mathematical Competence index, has a standard prediction value, and validation and reliability scores were significant.
\end{abstract}

Key words: Early math, cognition, assessment, number sense, kindergarden, primary school.

Correspondencia: José I. Navarro. Departamento de Psicología, Universidad de Cádiz, Campus Río San Pedro, C.P. 11510. Puerto Real-Cádiz (España). E-mail: jose.navarro@uca.es 
Un nivel de competencia matemática temprana (CMT) suficiente es un requisito para ser capaz de seguir una educación matemática formal y ha llamado la atención de los investigadores (Aunio, Hautamäki, Sajaniemi y Van Luit, 2009; Kroesbergen, Van Luit, Van Lieshout, Van Loosbroek y Van de Rijt, 2009; Van de Rijt, Van Luit y Pennings, 1999). La CMT determinada a partir de las tareas de conservación del número se basa en una compleja síntesis de operaciones de clasificación de objetos operando con el principio de relación de 1 a 1 y el de seriación. La conservación del número requiere además comprender sus aspectos cardinales y ordinales. Incluye también su aplicación a los procesos de conteo y a situaciones de la vida cotidiana en las que se necesita el número para resolver determinados problemas.

Ha habido un consenso en relación a las fases y las edades en las que se desarrolla el conocimiento matemático temprano (Fuson, 1988; Griffin, 2002). También se ha encontrado que los niños a la edad de 5 y 6 años pueden ser capaces de resolver tareas de conservación de números y de correspondencia haciendo uso del conteo (Gelman y Gallistel, 1978; Leslie, Gelman y Gallistel, 2008). La teoría y la práctica sobre el desarrollo y enseñanza de los conceptos matemáticos en los niños/as pequeños ha estado fuertemente influida por el modelo de operaciones lógicas de Piaget (Piaget y Szeminska, 1967) que incluye las nociones de clasificación, seriación y conservación. La posición piagetiana apunta una fuerte relación entre las operaciones lógicas y los conceptos numéricos y también con las operaciones aritméticas. Consecuente con este modelo, el papel del conteo verbal en el desarrollo de los conceptos numéricos y destrezas matemáticas ha sido considerado como una habilidad de segunda categoría (Payne y Huinker, 1993). Sin embargo, estudios más recientes han cuestionado el papel central de las operaciones lógicas, mostrando al mismo tiempo la importancia del conteo en el desarrollo matemático (Mayer, 2002). Otros trabajos, sin embargo, mantienen la importancia del desarrollo de las operaciones piagetianas para un adecuado conocimiento matemático (Baroody y Dowker, 2003).

El Test de Evaluación Matemática Temprana (TEMT) desarrollado en Holanda por Van de Rijt et al. (1999) está basado en la realización de tareas, y orientado a medir el nivel de competencia matemática temprana. Se ha desarrollado para $2^{\circ}$ y $3^{\circ}$ de educación infantil y $1^{\circ}$ y $2^{\circ}$ de educación primaria y ha sido baremado en varios países europeos. El test no está ligado a ningún método de enseñanza o aprendizaje de las matemáticas. Dispone de tres versiones paralelas de 40 ítems cada una. Consta de 8 tareas, divididas en grupos de 5. Tiene una puntuación máxima de 40 puntos (uno por cada ítem correcto).

Debe ser administrado individualmente. Con cualquiera de las versiones paralelas, el psicólogo escolar será capaz de llevar el seguimiento del desarrollo de la CMT de un alumno/a o un grupo. Comparando el resultado de un niño con un grupo 
normativo puede determinarse el nivel de CMT. Existe la posibilidad de aplicar otra de las versiones cuando nos interese verificar un valor no esperado en el test.

Los resultados del test en niños pequeños pueden estar determinados por las condiciones en las que se administra y de esta forma podrían ser inesperadamente altos o bajos. Para ver si este es el caso en el TEMT, puede administrarse una de las versiones paralelas unos días más tarde. Si esas circunstancias no influyeron de forma significativa en la primera administración, en el segundo pase el niño/a tendrá una puntación similar. Pero si esas condiciones específicas jugaron un papel importante en la primera administración, los resultados en la segunda quizás estén más cercanos a los esperados.

Además de la determinación del nivel de CMT, el evaluador puede ser también capaz, con la ayuda de las tres versiones del test, de asegurarse si el niño progresa durante el curso o con la metodología de enseñanza de la matemática utilizada. Al examinar los resultados de las versiones A, B o C al comienzo y al final del programa educativo, el profesor podrá averiguar qué mejora ha alcanzado el niño/a en su nivel de competencia matemática temprana. Los resultados obtenidos por el alumno/a sólo pueden compararse con los que ha obtenido anteriormente en el test por él mismo, o bien con las puntuaciones obtenidas por sus compañeros de la misma edad.

El objetivo fundamental de este estudio consistió en adaptar el test a la población española, de forma que los profesionales de la educación puedan tener un instrumento eficaz para detectar posibles problemas en el aprendizaje de las matemáticas al inicio de la escolaridad.

\section{METODO}

\section{Participantes}

Un total de 1.053 niños/as pertenecientes a 14 colegios públicos o concertados han participado en este estudio. 539 eran varones (51.2\%) y 514 mujeres ( $48.8 \%$ ) (tabla 1). Los participantes tenían una procedencia de clase media y clase media baja. Los cursos de escolarización iban desde educación infantil de 4 años hasta $2^{\circ}$ de primaria. Estuvieron distribuidos geográficamente en las provincias de Cádiz, Sevilla, Madrid y Salamanca.

\section{Instrumentos}

Los componentes evaluados por la prueba TEMT son los siguientes:

1. Conceptos de comparación. Este aspecto se refiere al uso de conceptos de comparación entre dos situaciones no equivalentes relacionados con el cardinal, el ordinal y la medida. Un ejemplo de ítem de este subtest es: "Aquí ves unos indios. Señala el indio que tiene menos plumas que éste que tiene su arco y sus flechas". 


\begin{tabular}{|c|c|c|c|c|}
\hline Edades (en años) & EI 4 años & EI 5 años & $1^{\circ} \mathrm{EP}$ & $2^{\circ} \mathrm{EP}$ \\
\hline $4,1-4,6$ & 114 & & & \\
\hline $4,7-5,0$ & 119 & 52 & & \\
\hline $5,1-5,6$ & 8 & 145 & & \\
\hline $5,7-6,0$ & & 147 & 11 & \\
\hline $6,1-6,6$ & & 22 & 117 & \\
\hline $6,7-7,0$ & & & 93 & 12 \\
\hline $7,1-7,6$ & & & 11 & 95 \\
\hline $7,7-8,0$ & & & & 107 \\
\hline
\end{tabular}

(EI: Educación Infantil; EP = Educación Primaria)

2. Clasificación. Se refiere al agrupamiento de objetos basándose en una o más características. Un ejemplo de ítem es: "Mira estos cuadrados. ¿Puedes señalar el que tiene cinco cuadrados pero ningún triángulo?". Con la tarea de clasificación se pretende conocer si los niños, basándose en la semejanza y en las diferencias, pueden distinguir entre objetos y grupos de ellos.

3. Correspondencia uno a uno. El niño debe ser capaz de establecer esta correspondencia entre diferentes objetos que son presentados simultáneamente. Una muestra de este subtest es el ítem 12: el evaluador le da al niño 15 cubos y le presenta un dibujo que representa las caras de dos dados con el patrón de puntos de 5 y 6 . "Yo he lanzado dos dados y he conseguido estos puntos. ¿Puedes darme la misma cantidad de cubos?".

4. Seriación. Se trata de averiguar si los niños son capaces de reconocer una serie de objetos ordenados. Los términos usados en esta tarea son: de mayor a menor, del más delgado al más grueso, de la más pequeña a la más grande. Por ejemplo: “Aquí ves unos cuadrados que tienen unos palitos. Señala el cuadrado donde los palitos están ordenados del más delgado al más grueso".

5. Conteo verbal. Se evalúa la secuencia numérica oral hasta el 20. Puede ser expresada contando hacia delante, hacia atrás y relacionándola con el aspecto cardinal y ordinal del número. Por ejemplo: "Cuenta desde el 9 hasta el 15".

6. Conteo estructurado. Contar un conjunto de objetos que son presentados con una disposición ordenada o desordenada. Los niños pueden señalar con el dedo los objetos que cuentan. Se trata de averiguar si son capaces de mostrar coordinación entre contar y señalar. Por ejemplo: El evaluador pone sobre la mesa un total de 20 cubos de forma desorganizada. El niño es requerido a que cuente todos los cubos.

7. Conteo resultante. El niño tiene que contar cantidades que son presentadas como colecciones estructuradas o no estructuradas y no se le permite señalar los objetos que tiene que contar. Un ejemplo es: Se le presenta al niño 15 cubos en tres filas de cinco cubos cada una con un espacio entre ellos y se le pregunta: "¿Cuántos cubos hay aquí?”. 
8. Conocimiento general de los números. Se refiere a la aplicación de la numeración a las situaciones de la vida diaria que son presentadas en forma de dibujo. Un ejemplo es: "Tú tienes 9 canicas. Pierdes 3 canicas. ¿Cuántas canicas te quedan?".

Cada uno de los ocho componentes del test tiene cinco ítems. Cada acierto se puntúa con 1 y los errores con 0 . La puntuación directa máxima que puede obtenerse es de 40. Los cuatro subtests primeros (Relacionales: ítems 1 a 20) evalúan habilidades de tipo piagetiano y los cuatro últimos (Numéricos: ítems 21 a 40) estiman habilidades numéricas de naturaleza cognitiva. La edad de administración del test se sitúa desde los 4 a los 7 años.

\section{Procedimiento}

Los autores del trabajo administraron el TEMT en su versión A o B de forma individual, dentro del centro escolar al que pertenecían los participantes y tras un estudio piloto. Completar el test lleva aproximadamente entre veinte y treinta minutos. Todos los ítems son presentados oralmente y los niños responden señalando en un material con dibujos o, en el caso de las tareas de contar y de numeración, manipulando pequeños cubos de madera del tipo unifix. Tres de los ítems requieren que el alumno/a use el lápiz para unir los objetos del dibujo presentado. La adaptación del test al castellano se hizo siguiendo las normas internacionales establecidas para la adaptación de material de pruebas de evaluación recogidas en Muñiz y Hambleton (1996).

\section{RESULTADOS}

La tabla 2 nos muestra los resultados medios obtenidos por los participantes que sirvieron como muestra normativa. A partir de esta tabla podemos conocer el nivel donde se encuentra un alumno según su edad y en función de la puntuación directa total obtenida en el TEMT. La tabla está distribuida en función de las agrupaciones de los subtests relacional y numérico. La última parte de la tabla recoge los valores (medias y desviación típìca) del total del TEMT según los grupos de edad.

\section{El nivel de competencia matemática (NCM)}

La puntuación directa del test es transformada en un valor denominado Nivel de Competencia Matemática (NCM). Este NCM indica el grado de dominio del conocimiento matemático temprano. Pero el NCM no da por sí mismo una información suficiente, es necesario hacer comparaciones de las puntuaciones del niño/a con los demás miembros de su clase o de su grupo de edad.

Para poder utilizar estos elementos de comparación, el test fue administrado a 1.053 niños y niñas de 4 a 8 años. De ellos se ha eliminado para el cálculo normativo 
a los menores de 4,07 años y a los mayores de 7,06 años, quedando una muestra de 832 casos distribuidos en 6 grupos. La tabla 3 recoge los límites de edad para los grupos (I-VI).

\begin{tabular}{cccc} 
Tabla 2. Media y desviación típica $(d t)$ obtenidas por los participantes en el test TEMT según la edad \\
\hline & Edad & Media & $d t$ \\
\hline & $4,1-4,6$ & 8,07 & 3,43 \\
Puntuación subtests relacionales & $4,7-5,0$ & 9,23 & 3,22 \\
& $5,1-5,6$ & 11,88 & 3,24 \\
& $5,7-6,0$ & 13,91 & 3,23 \\
& $6,1-6,6$ & 16,10 & 2,49 \\
& $6,7-7,0$ & 17,09 & 2,15 \\
& $7,1-7,6$ & 17,79 & 1,99 \\
Puntuación subtests numéricos & $7,7-8,0$ & 18,37 & 1,66 \\
\hline & $4,1-4,6$ & 2,42 & 2,99 \\
& $4,7-5,0$ & 3,34 & 3,12 \\
& $5,1-5,6$ & 6,50 & 4,20 \\
& $5,7-6,0$ & 9,20 & 4,77 \\
& $6,1-6,6$ & 13,58 & 3,46 \\
& $6,7-7,0$ & 14,97 & 3,64 \\
& $7,1-7,6$ & 16,81 & 2,82 \\
& $7,7-8,0$ & 17,59 & 2,25 \\
\hline & $4,1-4,6$ & 10,46 & 5,64 \\
& $4,7-5,0$ & 12,58 & 5,71 \\
& $5,1-5,6$ & 18,39 & 6,65 \\
& $5,7-6,0$ & 23,11 & 7,30 \\
& $6,1-6,6$ & 29,69 & 5,39 \\
& $6,7-7,0$ & 32,07 & 5,21 \\
& $7,1-7,6$ & 34,64 & 4,36 \\
& $7,7-8,0$ & 35,97 & 3,18 \\
\hline
\end{tabular}

Tabla 3. Edad de los grupos establecidos (I-VI), muestra utilizada en cada grupo

\begin{tabular}{cccc}
\multicolumn{4}{c}{ de edad (n) y porcentaje de la muestra en cada grupo } \\
\hline Grupo & Edad & $\mathrm{n}$ & $\%$ \\
\hline I & $4,07-5,00$ & 171 & 20,6 \\
II & $5,01-5,06$ & 153 & 18,4 \\
III & $5,07-6,00$ & 158 & 19,0 \\
IV & $6,01-6,06$ & 139 & 16,7 \\
V & $6,07-7,00$ & 105 & 12,6 \\
VI & $7,01-7,06$ & 106 & 12,7 \\
\hline Total & & 832 & 100 \\
\hline
\end{tabular}

Al aplicar la tabla de resultados normativa, la puntuación obtenida por cada niño/a se puede transformar en el NCM. De esta forma la puntuación obtenida en el NCM es comparada con las puntuaciones obtenidas por los otros niños del mismo grupo de edad. Los niveles han sido establecidos de la siguiente manera:

Nivel A: Muy bueno (comparable con el $25 \%$ de las puntuaciones más altas obtenidas por los niños de su grupo normativo). 
Nivel B: Bueno (comparable con el $25 \%$ de las puntuaciones por encima de la media obtenida por los niños de su grupo normativo).

Nivel C: Moderado (comparable con el $25 \%$ de las puntuaciones por debajo de la media obtenida por los niños de su grupo normativo).

Nivel D: Bajo (comparable con el $15 \%$ de las puntuaciones por debajo de la media obtenida por los niños de su grupo normativo).

Nivel E: Muy bajo (comparable con el $10 \%$ de las puntuaciones muy por debajo de la media obtenida por los niños de su grupo normativo).

Cuando ha sido establecido el nivel de un niño, el usuario de la prueba tiene una visión completa del Nivel de Competencia Matemática en comparación con su grupo normativo, conociéndose así el rango que ocupa en ese grupo. Los niños/as que obtienen el nivel A, por ejemplo, han alcanzado una puntuación que los iguala en su nivel de competencia del $25 \%$ superior del grupo. Y los que obtienen un nivel de competencia D, han sido superados por el 75 al $90 \%$ de los miembros de su grupo normativo. Por ejemplo, supongamos que Sergio, un niño de 53 meses, ha obtenido una puntuación directa total en el TEMT de 15 puntos que equivaldría a un nivel de competencia en el test de 23. Por la edad, estaría en el grupo normativo I y un nivel de Competencia Matemática B. Es decir, un nivel comparable con el $25 \%$ de las puntuaciones ligeramente por encima de la media obtenidas por los niños de su grupo normativo.

\begin{tabular}{|c|c|c|c|c|c|c|}
\hline $\mathrm{NCM}$ & I & II & III & IV & V & VI \\
\hline A & $>27$ & $>43$ & $>60$ & $>80$ & $>88$ & $>92$ \\
\hline B & $14-27$ & $29-43$ & $42-60$ & $64-80$ & $73-88$ & $81-92$ \\
\hline $\mathrm{C}$ & $5-13$ & $17-28$ & $32-41$ & $52-63$ & $61-72$ & $73-80$ \\
\hline D & $3-4$ & $10-16$ & $15-31$ & $43-51$ & $51-60$ & $66-72$ \\
\hline E & $<3$ & $<10$ & $<15$ & $<43$ & $<51$ & $<66$ \\
\hline
\end{tabular}

La fiabilidad del TEMT fue calculada a partir del Alfa de Cronbach, según se recoge en la tabla 5. En general se consideran aceptables coeficientes de fiabilidad superiores a 0,80. Los resultados obtenidos en el TEMT superan este valor. Se ha realizado el cálculo con todos lo participantes $(\mathrm{N}=1053)$ para los subtests relacionales, numéricos y el total de la prueba. El dato del coeficiente global que arroja un alfa de Cronbach $=0,88$ nos indica la calidad de la medida que permite el instrumento. Posiblemente el tamaño tan numeroso de la muestra permite conocer de forma más fidedigna las características del TEMT.

En la tabla 5 se muestra los errores típicos de medida (ETM) de cada subescala y del total del test, derivados a partir de los coeficientes de fiabilidad entendidos como consistencia interna según la siguiente fórmula: $S_{e}=S_{x} \sqrt{1-r_{x x}}$. 
Donde $S_{e}$ es el error típico de medida; $S_{x}$ es la desviación típica del TEMT; y $r_{x x}$ es el coeficiente de fiabilidad. El error típico de medida es un índice de incertidumbre asociado a la evaluación y se puede utilizar para establecer intervalos de confianza alrededor de una puntuación. Así, a partir de la puntuación obtenida por un niño/a en el TEMT en un momento concreto, podemos establecer un intervalo en el que se situará su auténtica puntuación con una cierta probabilidad de error. El conocimiento del intervalo de confianza, es muy útil para conocer el rango en el que se situará realmente la puntuación de un niño/a.

\begin{tabular}{|c|c|c|c|}
\hline & Alfa & ETM & Int. $90 \%$ \\
\hline Total del test & 0,88 & 1,61 & $\pm 2,65$ \\
\hline Subtest Relacional & 0,94 & 1,62 & $\pm 2,67$ \\
\hline Subtest Numéricos & 0,93 & 2,32 & $\pm 3,82$ \\
\hline Alfa de Cronbach general & 0,952 & & \\
\hline
\end{tabular}

Fiabilidad mediante formas paralelas. Un aspecto a tener en cuenta en la fiabilidad es conocer la estabilidad de las puntuaciones en el tiempo. Dado que en el caso de este test la maduración y el aprendizaje pueden afectar a los resultados, hemos administrado a un total de 127 participantes las versiones A y B del TEMT con un intervalo temporal de 8 meses. Los resultados se muestran en la tabla 6, considerando que son satisfactorios, mostrando la calidad técnica del instrumento.

\begin{tabular}{lccc}
\multicolumn{4}{c}{ Tabla 6. Fiabilidad mediante formas paralelas de las versiones A y B del TEMT (n = 127) } \\
\hline & $\begin{array}{c}\text { Subtest } \\
\text { Relacional } \\
\text { Forma B }\end{array}$ & $\begin{array}{c}\text { Subtest } \\
\text { Numéricos } \\
\text { Forma B }\end{array}$ & $\begin{array}{c}\text { Puntuación } \\
\text { Total } \\
\text { Forma B }\end{array}$ \\
\hline Subtest Relacional Forma A &, $505(* *)$ &, $509\left(^{* *}\right)$ &, $572(* *)$ \\
Subtest Numéricos Forma A &, $450(* *)$ &, $665\left(^{* *}\right)$ &, $649\left(^{* *}\right)$ \\
Puntuación Total Forma A &, $516(* *)$ &, $651\left(^{* *}\right)$ &, $671\left(^{* *}\right)$ \\
\hline$* *$ La correlación es significativa al nivel 0,01 (bilateral).
\end{tabular}

Validez de constructo. Realizando un análisis factorial exploratorio común se observa que cada una de las pruebas del TEMT evalúa destrezas matemáticas diferentes pero con una relación interna, bajo las cuales se encuentra una aptitud matemática general, que pudiéramos verificar como una capacidad destacada de la interacción de estas aptitudes. Al encontrar un factor lo suficientemente explicativo a partir de los pesos encontrados en las diversas subpruebas del TEMT, que se muestra en la tabla 7, se constata que el factor resultante del análisis explica el $69 \%$ de la varianza encontrada. Entendemos que la peculiaridad de las distintas capacidades asume la variabilidad restante. 
Validez Divergente. Aunque existe una cierta varianza común, la aplicación de los distintos subtests se justifica por la evaluación de las diferentes competencias matemáticas. Para analizar esto, se calcularon las inter correlaciones existentes entre las diferentes subpruebas del TEMT. Los resultados que se esperan en este caso son de correlaciones altas y significativas entre las diferentes subescalas, como se recoge en la tabla 8 . Nos indican que los resultados en algunas pruebas pueden predecirse a partir de los resultados de otras.

Tabla 7. Pesos factoriales de los subtests del TEMT

\begin{tabular}{cc}
\hline & Peso factorial \\
\hline Comparación & 0,655 \\
Clasificación & 0,714 \\
Correspondencia & 0,864 \\
Seriación & 0,841 \\
Conteo verbal & 0,906 \\
Conteo estructurado & 0,881 \\
Conteo resultante & 0,891 \\
Conocimiento general de los números & 0,868 \\
\hline
\end{tabular}

Tabla 8. Inter correlaciones de las diferentes subescalas básicas del TEMT

\begin{tabular}{|c|c|c|c|c|c|c|c|c|c|}
\hline & $\mathrm{COM}$ & CLAS & COR & $\mathrm{SE}$ & VE & ES & $\mathrm{RE}$ & $\mathrm{CON}$ & TOTAL \\
\hline $\mathrm{COM}$ & 1 & $0,486(* *)$ & $0,344(* *)$ & 0,170 & $0,282(* *)$ & $0,369(* *)$ & $0,205(*)$ & $0,321(* *)$ & $0,496(* *)$ \\
\hline CLAS & & 1 & $0,423(* *)$ & $0,272(* *)$ & $0,413(* *)$ & $0,327(* *)$ & $0,249(* *)$ & $0,332(* *)$ & $0,578(* *)$ \\
\hline COR & & & 1 & $0,453(* *)$ & $0,589(* *)$ & $0,584(* *)$ & $0,525(* *)$ & $0,639(* *)$ & $0,811(* *)$ \\
\hline SER & & & & 1 & $0,427(* *)$ & $0,270(* *)$ & $0,302(* *)$ & $0,431(* *)$ & $0,612(* *)$ \\
\hline VER & & & & & 1 & $0,607(* *)$ & $0,645(* *)$ & $0,632(* *)$ & $0,828(* *)$ \\
\hline EST & & & & & & 1 & $0,604(* *)$ & $0,582(* *)$ & $0,768(* *)$ \\
\hline RES & & & & & & & 1 & $0,539(* *)$ & $0,727(* *)$ \\
\hline $\mathrm{CON}$ & & & & & & & & 1 & $0,806(* *)$ \\
\hline TOTAL & & & & & & & & & 1 \\
\hline \multicolumn{10}{|c|}{$\begin{array}{l}\text { ** La correlación es significativa al nivel 0,01 (bilateral). * La correlación es significante al nivel } 0,05 \\
\text { (bilateral). COM = COMPARACIÓN; CLAS = CLASIFICACIÓN; COR = CORRESPONDENCIA; } \\
\text { SE = SERIACIÓN; VE = CONTEO VERBAL; EST = CONTEO ESTRUCTURADO; } \\
\text { RE = CONTEO RESULTANTE; CON = CONOCIMIENTO GENERAL DE LOS NÚMEROS; } \\
\text { TOTAL = PUNTUACIÓN TOTAL EN EL TEST. }\end{array}$} \\
\hline
\end{tabular}

Validez Concurrente. Se ha calculado la validez concurrente del TEMT comparando sus resultados con los obtenidos por una submuestra de 127 niños/as en el Tedi-Math (Van Nieuwenhoven, Noel, y Grégoire, 2005) que evalúa diversos componentes de la competencia numérica (habilidades de conteo y enumeración, conocimientos del sistema de numeración arábigo y verbal, estimación de cantidades, operaciones aritméticas y competencias lógicas). En este test, el desarrollo operatorio se evalúa con cinco pruebas. Algunas de las pruebas siguen el procedimiento clásico propuesto por Piaget, otras son novedosas porque evalúan el desarrollo lógico a través de situaciones numéricas. Han sido elaboradas para reducir al máximo la influencia de 
variables no deseadas. Los subtests del Tedi-Math presentan una fiabilidad mayor de 0,80. En las pruebas lógicas el alfa de Cronbach es de 0,93. La tabla 9 recoge las correlaciones establecidas entre ambas pruebas. Todas las comparaciones de interés entre los dos tests resultan significativas, especialmente la puntuación total del TEMT con la puntuación total del Tedi-Math $(r=0,689, p<0,01)$.

Tabla 9. Correlaciones entre puntuaciones directas (PD) obtenidas en el TEMT comparando sus resultados con los obtenidos por 127 niños/as en el TEDI-MATH

\begin{tabular}{|c|c|c|c|c|c|c|c|c|c|}
\hline & $\mathrm{COM}$ & CLAS & COR & SE & VE & ES & $\mathrm{RE}$ & NU & TEMT \\
\hline $\mathrm{CO}$ & $0,291(* *)$ & $0,358(* *)$ & $0,529(* *)$ & $0,328(* *)$ & $0,665(* *)$ & $0,544(* *)$ & $0,518(* *)$ & $0,477(* *)$ & $0,659(* *)$ \\
\hline NU & $0,206\left(^{*}\right)$ & $0,221(*)$ & $0,257(* *)$ & 0,150 & $0,335(* *)$ & $0,360(* *)$ & $0,361(* *)$ & $0,270(* *)$ & $0,378(* *)$ \\
\hline $\mathrm{AR}$ & 0,090 & $-0,011$ & $0,251(* *)$ & $0,182(*)$ & $0,226\left(^{*}\right)$ & $0,179\left(^{*}\right)$ & 0,084 & $0,198(*)$ & $0,205(*)$ \\
\hline OR & $0,260(* *)$ & 0,095 & $0,251(* *)$ & 0,166 & $0,175\left(^{*}\right)$ & $0,246(* *)$ & $0,214\left(^{*}\right)$ & $0,206\left(^{*}\right)$ & $0,278(* *)$ \\
\hline LOG & 0,174 & $0,189\left(^{*}\right)$ & $0,360(* *)$ & $0,388(* *)$ & $0,389(* *)$ & $0,300(* *)$ & $0,402(* *)$ & $0,317(* *)$ & $0,448(* *)$ \\
\hline IMA & $0,368(* *)$ & $0,414(* *)$ & $0,411(* *)$ & 0,139 & $0,371(* *)$ & $0,372(* *)$ & $0,283(* *)$ & $0,401(* *)$ & $0,472(* *)$ \\
\hline TA & $0,193\left(^{*}\right)$ & $-0,034$ & 0,150 & 0,159 & $0,245(* *)$ & $0,220\left(^{*}\right)$ & $0,193\left(^{*}\right)$ & $0,260(* *)$ & $0,249(* *)$ \\
\hline $\begin{array}{r}\text { TEDI } \\
\text { MATH } \\
\end{array}$ & $0,377(* *)$ & $0,360(* *)$ & $0,559(* *)$ & $0,350(* *)$ & $0,628(* *)$ & $0,582(* *)$ & $0,547(* *)$ & $0,523(* *)$ & $0,689(* *)$ \\
\hline
\end{tabular}

** La correlación es significativa al nivel 0,01 (bilateral);* La correlación es significativa al nivel 0,05 (bilateral). ${ }^{* *}$ La correlación es significativa al nivel 0,01 (bilateral). * La correlación es significativa al nivel 0,05 (bilateral). $\mathrm{COM}=\mathrm{COMPARACIÓN}$; CLAS $=$ CLASIFICACIÓN; COR $=$ CORRESPONDENCIA; $\mathrm{SE}=$ SERIACIÓN; VE = CONTEO VERBAL; EST = CONTEO ESTRUCTURADO; RE = CONTEO RESULTANTE; CON = CONOCIMIENTO GENERAL DE LOS NÚMEROS; TEMT = PUNTUACIÓN TOTAL EN EL TEST TEMT; $C O=$ CONTAR; UN = NUMERAR; UN = SISTEMA NUMÉRICO ARÁNIGO; OR = SISTEMA NUMÉRICO ORAL; LOG = OPERACIONES LOGICAS; IMA = OPERACIONES CON APOYO DE IMÁGENES; TA = ESTIMACION DEL TAMAÑO; TEDI-MATH $=$ PUNTUACIÓN TOTAL EN EL TEST TEDI-MATH

Validez Predictiva. Permite comprobar si los resultados de la prueba inicial predicen los resultados en otra prueba o criterio externo evaluado tras un período de tiempo. Entre los criterios más frecuentes utilizados para determinar la validez predictiva destacan la actuación en práctica, las calificaciones escolares, los niveles de inteligencia o el ejercicio profesional durante un tiempo (García-Hoz y Pérez-Juste, 1984). Para calcular la validez predictiva del TEMT, se ha utilizado una muestra de 50 participantes que fueron evaluados con el test al empezar el tercer curso de educación infantil (5 años). Ha sido evaluada también con una prueba de matemáticas de carácter curricular al terminar segundo de Educación Primaria (7 años de edad de promedio). La prueba es utilizada para evaluar los conocimientos matemáticos del alumnado al terminar el primer ciclo de la Educación Primaria. Para hallar estos índices de validez se ha utilizado las puntuaciones obtenidas en numeración, cálculo y resolución de problemas. 
Tabla 10. Correlaciones significativas entre los subtests del TEMT y las puntuaciones obtenidas en una prueba curricular de Matemáticas (al terminar $2^{\circ}$ de educación primaria)

\begin{tabular}{lc}
\hline & CORRELACIONES \\
\hline Clasificación &, $473(* *)$ \\
Correspondencia &, $\left.464 *^{* *}\right)$ \\
Seriación &, $406\left(^{* *}\right)$ \\
Conteo verbal &, $475(* *)$ \\
Conteo estructurado &, $585\left(^{* *}\right)$ \\
Resultante &, $478(* *)$ \\
Conocimiento general de los números &, $453(* *)$ \\
Total subtests relacionales &, $689\left(^{* *}\right)$ \\
Total subtests numéricos &, $633(* *)$ \\
\hline$* *$ La correlación es significativa al nivel $0,01($ bilateral)
\end{tabular}

** La correlación es significativa al nivel 0,01 (bilateral).

Considerando globalmente la validez predictiva del TEMT, los coeficientes de correlación son significativos. Podemos concluir, por tanto, que el TEMT posee una adecuada validez predictiva, de forma que puede predecirse el rendimiento futuro (dos años después) en matemáticas (numeración, cálculo y resolución de problemas) del alumnado que se escolariza en educación infantil

\section{DISCUSION}

El TEMT resulta un test de evaluación del conocimiento numérico temprano fácil de administrar y válido para conocer el nivel de competencia numérica de los alumnos de infantil y primer ciclo de primaria. Permite tener una visión básica de las posibles fortalezas y debilidades del aprendizaje inicial de la matemática, facilitando la labor del profesor para realizar actividades de refuerzo en las áreas que aparezcan especialmente deficitarias, de acuerdo con las puntuaciones obtenidas en la prueba, pudiendo ser de gran ayuda al psicólogo escolar. Resulta particularmente importante en la administración de este test, la observación de las estrategias utilizadas por los niños durante la administración del mismo. El administrador de la prueba tiene que tener en cuenta esto, puesto que la información así registrada será de suma utilidad en cuanto al análisis de los procesos de resolución de problemas puestos en funcionamiento por los alumnos/as evaluados. Por ejemplo, en el ítem A5 (ver figura 1) se le pide al niño que señale la caja que tiene menos bolas (solución correcta: A); algunos niños responden señalando directamente la solución A; otros en cambio, cuentan las bolas de cada caja antes de responder. Otro ejemplo podemos encontrarlo en el ítem A15, en el que se le pide al niño que señale el cuadrado que tiene tantos puntos como globos; algunos niños con estrategias más eficientes dicen "cinco, cinco y cinco, 15: esta es la solución, 15"; en cambio, otros niños de su mismo grupo de edad tienen que ir contando punto a punto en cada uno de los cuadrados. En el ítem A35, se les pide que sumen 5 y 7 cubos sin tenerlos a la vista; el repertorio de respuestas es también muy variado. Por ejemplo, 
los alumnos/as con estrategias más avanzadas responden "12" (recuperación desde la memoria), sumar cinco a siete (contar a partir del mayor), o contar todos de uno en uno, hasta 12). En los tres casos la respuesta es correcta, pero el registrar estas observaciones nos permite conocer mejor qué tipo de estrategias más o menos avanzadas utiliza para la búsqueda de la respuesta.
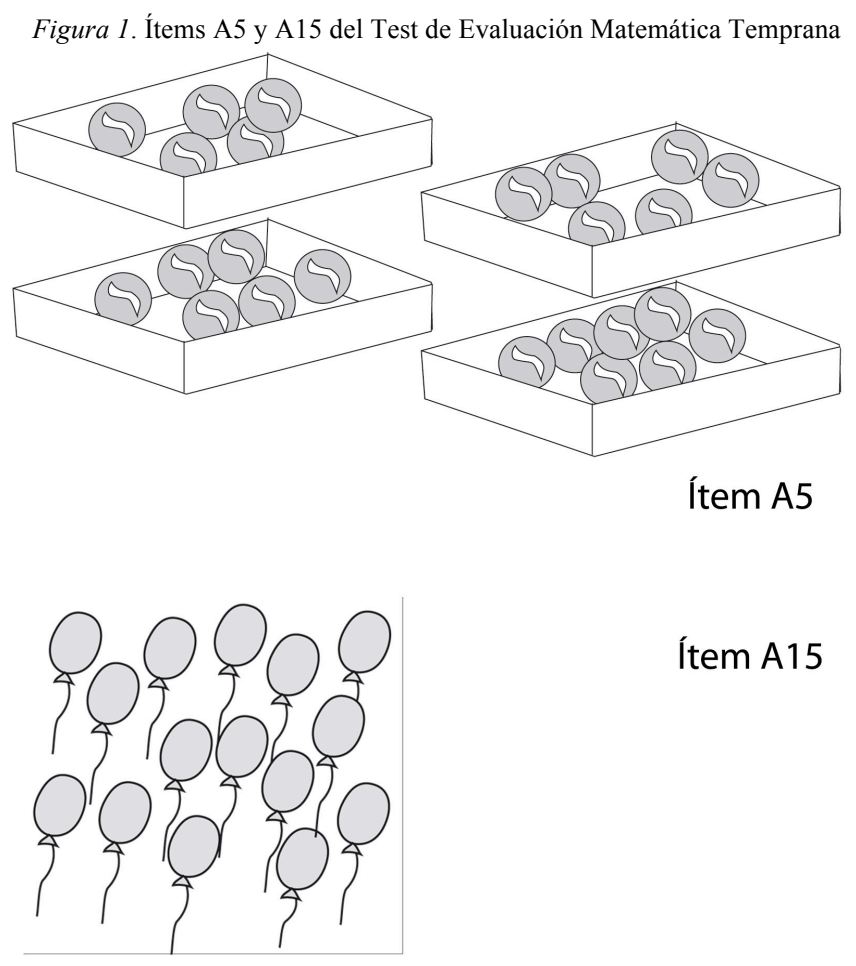

Ítem A15

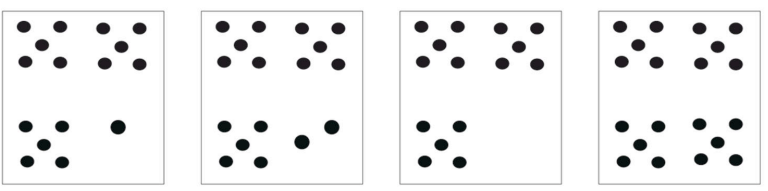

En realidad el TEMT no sólo permite conocer el nivel de competencia matemática, sino que el estudio de los procedimientos de resolución de las distintas tareas del test, puede ayudar a dilucidar los procesos y estrategias en los que el alumno/a se basa para resolver las actividades. El conocimiento de los parámetros cuantitativos y cualitativos de la ejecución matemática permite intervenir sobre el alumnado que presente dificultades de aprendizaje, teniendo en cuenta que el TEMT 
puede usarse como una prueba diagnóstica rápida para ser usada por los psicólogos escolares

Agradecimientos

Este trabajo fue desarrollado con los proyectos de investigación: SEJ200506881, SEJ2007-62420/EDUC y por el PAI.

\section{REFERENCIAS}

Aunio, P., Hautamäki, J., Sajaniemi, N. \& Van Luit, J.E.H. (2009). Early numeracy in lowperforming young children. British Educational Research Journal, 1, 25-46.

Fuson, K. (1988). Children's Counting and Concepts of Number. New York: Springer-Verlag.

García-Hoz, V. y Pérez-Juste, R. (1984). La investigación del profesor en el aula. Madrid: Escuela Española.

Gelman, R. \& Gallistel, C.R. (1978). The child's understanding of number. Cambridge: HUP.

Griffin, S. (2002). The development of math competence in the preschool and early school years: Cognitive foundations and instructional strategies. En J. Royer (Ed.), Mathematical cognition (pp. 1-32). Greenwich, CT: Information Age Publishing.

Kroesbergen, E.H., Van Luit, J.E.H., Van Lieshout, E.C.D.M., Van Loosbroek, E. \& Van de Rijt, B.A.M. (2009). Individual differences in early numeracy: The role of executive functions and subitizing. Journal of Psychoeducational Assessment. http://jpa.sagepub.com/cgi/content/abstract/0734282908330586v1

Leslie, A.M., Gelman, R. \& Gallistel, C.R. (2008). The generative basis of natural number concepts. Trends in Cognitive Sciences, 12, 213-218.

Muñiz, J. y Hambleton, R. K. (1996). Directrices para la traducción y adaptación de los tests. Papeles del Psicólogo, 66, 63-70.

Van de Rijt, B.A.M., Van Luit, J.E.H. \& Pennings, A.H. (1999). The construction of the Utrech Early Mathematical Competence Scales. Educational and Psychological Measurement, 59, 289-309.

Van Nieuwenhoven, C., Noel, M.P. \& Grégoire, J. (2005). Tedi-Math. Madrid: TEA.

Recibido: 18 de febrero de 2009

Modificaciones: 9 de abril de 2009

Aceptado: 23 de abril de 2009 